\title{
Non-Standard Neutrino Interactions
}

\author{
Enrique Fernandez Martinez* \\ CERN \\ E-mail: Enrique.Fernandez.Martinez@cern.ch
}

\begin{abstract}
We review the present model independent bounds on neutrino non-standard interactions both at neutrino production and detection and in its interactions with matter. This is interesting in view of the latest anomalies reported by the MINOS and MinoBooNE collaborations which could be accommodated with relatively large non-standard interactions. For matter non-standard interactions the direct bounds are rather weak. However, matter non-standard interactions are related by gauge invariance to the production and detection ones as well as to flavour changing processes involving charged leptons. Taking into account these relations much stronger bounds of at least $\mathscr{O}\left(10^{-3}\right)$ can be derived unless significant fine tunings are implemented. Testing non-standard interactions at this level is challenging but still feasible at proposed facilities such as MINSIS or the Neutrino Factory.
\end{abstract}

The Xth Nicola Cabibbo International Conference on Heavy Quarks and Leptons, October 11-15, 2010

Frascati (Rome) Italy

\footnotetext{
* Speaker.
} 


\section{Introduction}

The formalism of non-standard neutrino interactions (NSI) is a very widespread and convenient way of parameterizing the effects of new physics in neutrino oscillations [1,2]. Even though present data constrain NSI to be a subleading effect in neutrino oscillation experiments, the possibility of their eventual detection or interference with neutrino oscillations has triggered a considerable interest in the community. Moreover, the recent results from the antineutrino runs at the MINOS [3] and MiniBooNE [4] experiments show anomalies with respect to the standard, three-neutrino picture that could be accommodated through $\mathscr{O}\left(10^{-1}-10^{-2}\right)$ NSI $[5,6,7]$. It is a common practice to study NSI in matter since the constraints on the NSI affecting production and detection processes are typically stronger $[8,9,10]$. Here we will review the present bounds on NSI and discuss the possibility of saturating them so as to accommodate the results from MINOS or MiniBooNE.

\section{CC-like NSI}

In this section we discuss NSI for source and detector charged-current processes, we will refer to these as charged-current-like NSI. Leptonic NSI are given by the effective Lagrangian density

$$
\mathscr{L}_{\mathrm{NSI}}^{\ell}=-2 \sqrt{2} G_{F} \varepsilon_{\gamma \delta}^{\alpha \beta P}\left[\bar{\ell}_{\alpha} \gamma^{\mu} P \ell_{\beta}\right]\left[\bar{v}_{\gamma} \gamma_{\mu} P_{L} v_{\delta}\right],
$$

where $G_{F}$ is the Fermi constant and $P$ is either $P_{L}$ or $P_{R}$ and, due to Hermiticity, $\varepsilon_{\gamma \delta}^{\alpha \beta P}=\varepsilon_{\delta \gamma}^{\beta \alpha P *}$. For NSI at neutrino production via muon decay $\alpha=\mu$ and $\beta=e$. Notice that $\alpha=\beta=e$ would instead correspond to NSI with electrons in matter.

In a similar fashion, the charged-current-like NSI with quarks are given by

$$
\mathscr{L}_{\mathrm{NSI}}^{q}=-2 \sqrt{2} G_{F} \varepsilon_{\alpha \beta}^{q q^{\prime} P} V_{q q^{\prime}}\left[\bar{q} \gamma^{\mu} P q^{\prime}\right]\left[\bar{\ell}_{\alpha} \gamma_{\mu} P_{L} v_{\beta}\right]+\text { h.c. }
$$

where $q$ is an up-type and $q^{\prime}$ is a down-type quark. Only $q=u$ and $q^{\prime}=d$ are of practical interest for neutrino oscillations, due to their contributions to charged-current interactions with pions and nuclei. In Ref. [10] bounds on these production and detection NSI were derived from constraints on the comparison of different measurements of $G_{F}$ : through $\mu$ decay (affected by leptonic NSI of Eq. (2.1)), $\beta$ decays (affected by quark NSI of Eq. (2.2)) and the kinematic measurements of the masses of the gauge bosons $M_{Z}$ and $M_{W}$ (not affected by neutrino NSI). Universality tests of $G_{F}$ from $\pi$ and $\tau$ decays were also considered. One of the most striking effects of production and detection NSI in neutrino oscillation experiments is the existence of "zero distance" effects. Indeed, in presence of the NSI described in Eqs. (2.1) and (2.2), the neutrino produced or detected in association with a charged fermion need not share its flavour. It is then possible to observe flavour transitions at the detector without the requirement of a long baseline for neutrinos to oscillate. This "zero distance" effect could have led to signals at very short baseline neutrino oscillation experiments such as KARMEN or NOMAD. These results were also considered in Ref. [10] when deriving bounds on production and detection NSI. Finally, some NSI operators such as $\varepsilon_{\mu e}^{u d L}$ mix at the loop level with flavour changing charged lepton operators that could induce $\mu$ to $e$ conversion in nuclei. From this mixing the expected rate of this process can be related to the size of $\varepsilon_{\mu e}^{u d L}$ 
and additional bounds can be derived. Ref. [10] contains a table with all the bounds derived on the NSI parameters through the processes described. In order to derive those bounds only one NSI parameter was switched on at a time in order to avoid the relaxation of the bounds through cancellations among different parameters. Here we present a summary of the most stringent bounds derived:

$$
\begin{aligned}
\left|\varepsilon_{\alpha \beta}^{\mu e}\right|< & \left(\begin{array}{ccc}
0.025 & 0.030 & 0.030 \\
0.025 & 0.030 & 0.030 \\
0.025 & 0.030 & 0.030
\end{array}\right), \\
\left|\varepsilon_{\alpha \beta}^{u d}\right|< & \left(\begin{array}{ccc}
0.041 & 0.025 & 0.041 \\
1.8 \cdot 10^{-6} & 0.078 & 0.013 \\
0.026 & & \\
0.087 & 0.013 & 0.13 \\
0.12 & 0.018 &
\end{array}\right) .
\end{aligned}
$$

Whenever two values are quoted, the upper value refers to left-handed NSI and the lower to righthanded NSI. These bounds stem from the direct effect of the corresponding effective operator and are rather week. However, Eqs. (2.1) and (2.2) are not gauge invariant and can be related to flavour changing processes involving charged leptons when promoting the neutrino fields to full lepton doublets which lead to much tighter constraints. As we will discuss in the following, these constraints can be evaded by particular $d=6$ operators that select the neutrino fields in the lepton doublets in the case of purely leptonic NSI. For production and detection NSI involving quarks at the $d=6$ operator level, the only direct way of generating them involves inducing the corresponding NSI in the charged lepton sector as well. Several operators with different chirality structures can be introduced for this task and it is conceivable that cancellations amongst them can enhance neutrino NSI while suppressing the corresponding charged lepton processes. This was studied in detail in Ref. [11] for processes involving tau flavour violation at production and detection processes, which can lead to clean tau appearance signals at near detectors. The results show that the combination of different tau decays into mesons can constrain all possible chirality structures and the coefficients of the corresponding $d=6$ operators are all found to be $\mathscr{O}\left(10^{-4}\right)$ when compared to $G_{F}$. This makes their detection rather challenging, but not impossible. Indeed, certain operators can have enhanced contributions to some processes with respect to the SM. For example, while neutrino production via pion decay is chirality suppressed in the SM, operators with a pseudoscalar structure will have an enhanced contribution. These NSI would receive a $m_{\pi}^{2} /\left(\left(m_{u}+m_{d}\right) m_{\mu}\right)$ enhancement which could lead to $v_{\tau}$ appearance at production at a probability level of $7.9 \cdot 10^{-5}$ despite the $\mathscr{O}\left(10^{-4}\right)$ coefficient bound in the corresponding operator. This could potentially be tested at an OPERA-like detector capable of tau identification situated at a short baseline from a neutrino beam from pion decay. MINSIS [12] constitutes such a proposal for a detector at $1 \mathrm{~km}$ from the NuMI beam and could test the tau appearance probability at the $10^{-6}$ level or better.

\section{NC-like NSI}

We will now also review the current status of the bounds on NSI matter effects, or neutral- 
current-like NSI defined as

$$
\mathscr{L}_{\mathrm{NSI}}^{M}=-2 \sqrt{2} G_{F} \varepsilon_{\alpha \beta}^{f P}\left[\bar{f} \gamma^{\mu} P f\right]\left[\bar{v}_{\alpha} \gamma_{\mu} P_{L} v_{\beta}\right]
$$

where $f=e, u, d$. This type of NSI is the most extensively studied in the literature, since the constraints on the charged-current-like NSI are generally stronger. Indeed, the bounds from Refs. [13, $14,15,16]$, but discarding the loop constraints on $\varepsilon_{e \mu}^{f P}$ given the discussion in [17], result in the following $\mathscr{O}\left(10^{-1}\right)$ constraints:

$$
\begin{aligned}
& \left|\varepsilon_{\alpha \beta}^{e}\right|<\left(\begin{array}{ccc}
0.06 & 0.10 & 0.4 \\
0.14 & & 0.27 \\
0.10 & 0.03 & 0.10 \\
0.4 & 0.10 & 0.16 \\
0.27 & & 0.4
\end{array}\right), \\
& \left|\varepsilon_{\alpha \beta}^{u}\right|<\left(\begin{array}{ccc}
1.0 & 0.05 & 0.5 \\
0.7 & & \\
0.05 & 0.003 & 0.05 \\
& 0.008 & \\
0.5 & 0.05 & 1.4 \\
0 & & 3
\end{array}\right) \text {, } \\
& \left|\varepsilon_{\alpha \beta}^{d}\right|<\left(\begin{array}{ccc}
0.3 & 0.05 & 0.5 \\
0.6 & & \\
0.05 & 0.003 & 0.05 \\
& 0.015 & \\
0.5 & 0.05 & 1.1 \\
& & 6
\end{array}\right) \text {. }
\end{aligned}
$$

Again, the operator in Eq. (3.1) is not gauge invariant and the simplest gauge invariant extension of Eq. (3.1) promoting the left-handed fields to full SU(2) doublets would lead to flavour changing operators with charged leptons, allowing to derive much stronger bounds than the ones showed here. In Refs. $[18,19]$ extensions of the SM leading to neutrino NSI in matter but avoiding their charged current counterparts were studied both through $d=6$ and $d=8$ operators.

Only two examples of SM extensions giving rise to matter NSI but avoiding their charged lepton counterpart exist at $d=6$. The most direct one involves an antisymmetric 4-lepton operator, generated from the exchange of virtual singly charged scalar fields. In the second possibility NSI are induced through the dimension 6 operator modifying the neutrino kinetic terms, generated by the exchange of virtual fermionic singlets. The latter operator generates the NSI in an indirect way, i.e. after canonical normalisation of the neutrino kinetic terms.

In the first case, NSI with electrons in matter appear through the operator [20, 21, 22]

$$
\mathscr{L}_{N S I}^{d=6, a s}=c_{\alpha \beta \gamma \delta}^{d=6, a s}\left(\overline{L^{c}} \alpha i \sigma_{2} L_{\beta}\right)\left(\bar{L}_{\gamma} i \sigma_{2} L_{\delta}^{c}\right),
$$

which is generated after integrating out a charged scalar SU(2) singlet coupling to the SM lepton doublets:

$$
\mathscr{L}_{\text {int }}^{S}=-\lambda_{\alpha \beta}^{i} \bar{L}_{\alpha}^{c} i \sigma_{2} L_{\beta} S_{i}=\lambda_{\alpha \beta}^{i} S_{i}\left(\bar{\ell}_{\alpha}^{c} P_{L} v_{\beta}-\bar{\ell}_{\beta}^{c} P_{L} v_{\alpha}\right)
$$


Integrating out the heavy scalars $S_{i}$ generates the dimension 6 operator of Eq. (3.3) at tree level. We find that, given the antisymmetric flavour coupling of Eq. (3.4), the only NSI induced are those between $v_{\mu}$ and $v_{\tau}$ with electrons in matter. Bounds on these NSI can be derived from $\mu$ and $\tau$ decays:

$$
\begin{aligned}
& \left|\varepsilon_{\mu \mu}^{m, e_{\mathrm{L}}}\right|<8.2 \cdot 10^{-4}, \\
& \left|\varepsilon_{\tau \tau}^{m, e_{\mathrm{L}}}\right|<8.4 \cdot 10^{-3}, \\
& \left|\varepsilon_{\mu \tau}^{m, e_{\mathrm{L}}}\right|<1.9 \cdot 10^{-3} .
\end{aligned}
$$

NSI in production and detection are also induced with similar strengths.

The second realization of NSI at $d=6$ is via the dimension 6 operator

$$
\mathscr{L}_{\text {kin }}^{d=6}=-c_{\alpha \beta}^{d=6, k i n}\left(\bar{L}_{\alpha} \cdot H^{\dagger}\right) i \not \supset\left(H \cdot L_{\beta}\right),
$$

which induces non-canonical neutrino kinetic terms [23, 24, 25] though the vev of the Higgs field. After diagonalising and normalising the neutrino kinetic terms, a non-unitary lepton mixing matrix is produced from this operator and hence non-standard matter interactions as well as related nonstandard interactions at the source and detector are induced. The tree level generation of this operator, avoiding a similar contribution to charged leptons that would lead to flavour changing neutral currents, requires the introduction of SM-singlet fermions which couple to the Higgs and lepton doublets via the Yukawa couplings (see e.g. [26]). Electroweak decays set the following bounds on the operator of Eq. (3.8) due to the effects a deviation from unitarity of the mixing matrix would imply $[27,25,18]$ :

$$
\frac{v^{2}}{2}\left|c_{\alpha \beta}^{d=6, k i n}\right|<\left(\begin{array}{ccc}
4.0 \cdot 10^{-3} & 1.2 \cdot 10^{-4} & 3.2 \cdot 10^{-3} \\
1.2 \cdot 10^{-4} & 1.6 \cdot 10^{-3} & 2.1 \cdot 10^{-3} \\
3.2 \cdot 10^{-3} & 2.1 \cdot 10^{-3} & 5.3 \cdot 10^{-3}
\end{array}\right) .
$$

These bounds can be translated to constraints in the matter NSI that would be induced by a nonunitary mixing:

$$
\left|\varepsilon_{\alpha \beta}\right|<\frac{v^{2}}{4}\left|\left(\frac{n_{n}}{n_{e}}-\delta_{\alpha e}-\delta_{e \beta}\right) c_{\alpha \beta}^{d=6, k i n}\right|
$$

Since the ratio of the neutron to electron density $\frac{n_{n}}{n_{e}}$ is in general close to 1 , this implies that the bounds on $\left|\varepsilon_{e \mu}\right|$ and $\left|\varepsilon_{e \tau}\right|$ are significantly stronger than the bounds on the individual $\varepsilon_{\alpha \beta}^{f}$. For the main constituents of the Earth's crust and mantle the factor $\frac{n_{n}}{n_{e}}-1$ means an additional suppression of two orders of magnitude of the NSI coefficient [18]. Thus, even if two $d=6$ possibilities exist to induce neutrino NSI while avoiding the corresponding charged lepton NSI, they cannot saturate the mild direct bounds from Eqs. (2.3) and (3.2) and stronger $\mathscr{O}\left(10^{-3}\right)$ bounds apply.

In Refs. $[18,19]$ the generation of matter NSI avoiding similar operators involving charged leptons was studied through $d=8$ operators. In [18] the analysis was restricted to new physics realizations that did not involve cancellations between diagrams involving different messenger particles. Under those conditions only three examples were found that involved the same mediators than the $d=6$ realizations, that is, either fermion singlets or singly charged scalar doublets. The 
bounds derived for the $d=6$ realizations can thus be translated also to the $d=8$ operators and, even if charged fermion NSI are avoided, no large neutrino NSI are obtained. In [19] it was shown that, allowing cancellation between diagrams involving different messengers, large neutrino NSI could be obtained by tunning away all other dangerous contributions to the charged lepton sector, but the high operator dimension required and the precise cancellation make this option rather contrived and no model has been proposed that can realize it.

\section{Conclusion}

We have reviewed the present model independent bounds on NSI. We find that production and detection NSI are bounded to be $<\mathscr{O}\left(10^{-2}\right)$. Conversely the bounds on matter NSI are around one order of magnitude weaker. Saturating these mild direct bounds can lead to large signals at neutrino experiments even accommodating the observed anomalies in the MINOS and MiniBooNE results. Matter NSI are however related to production and detection NSI and to flavour changing operators for charged leptons through gauge invariance. Exploring gauge invariant realizations we find that, barring fine tunings as the ones discussed in [19], gauge invariant NSI are constraint to be $\mathscr{O}\left(10^{-3}\right)$. This precludes any simple interpretation of the MINOS and MiniBooNE anomalies in terms of NSI and makes them challenging to probe at present $[12,11]$ or even future neutrino oscillation facilities such as the Neutrino Factory [28, 29, 30].

\section{References}

[1] L. Wolfenstein. Neutrino oscillations in matter. Phys. Rev. D17 2369, 1978.

[2] S. P. Mikheyev and A. Y. Smirnov. Resonance enhancement of oscillations in matter and solar neutrino spectroscopy. Sov. J. Nucl. Phys. $\mathbf{4 2}$ 913, 1985.

[3] P. Vahle. Results from MINOS. Neutrino 2010, XXIV International Conference on Neutrino Physics and Astrophysics .

[4] R. Van de Water. New Antineutrino Oscillation Results from MiniBooNE. Neutrino 2010, XXIV International Conference on Neutrino Physics and Astrophysics .

[5] W. A. Mann, D. Cherdack, W. Musial and T. Kafka. Apparent multiple $\Delta m_{32}^{2}$ in muon anti-neutrino and muon neutrino survival oscillations from non-standard interaction matter effect 2010. 1006.5720 .

[6] E. Akhmedov and T. Schwetz. MiniBooNE and LSND data: non-standard neutrino interactions in a $(3+1)$ scheme versus $(3+2)$ oscillations. JHEP 10 115, 2010. 1007.4171.

[7] J. Kopp, P. A. N. Machado and S. J. Parke. Interpretation of MINOS data in terms of non-standard neutrino interactions 2010. 1009.0014.

[8] Y. Grossman. Nonstandard neutrino interactions and neutrino oscillation experiments. Phys. Lett. B359 141, 1995. hep-ph/9507344.

[9] M. C. Gonzalez-Garcia, Y. Grossman, A. Gusso and Y. Nir. New CP violation in neutrino oscillations. Phys. Rev. D64 096006, 2001. hep-ph/ 0105159.

[10] C. Biggio, M. Blennow and E. Fernandez-Martinez. General bounds on non-standard neutrino interactions. JHEP 08 090, 2009. 0907.0097. 
[11] S. Antusch, M. Blennow, E. Fernandez-Martinez and T. Ota. New physics searches at near detectors of neutrino oscillation experiments. JHEP 06 068, 2010. 1005.0756.

[12] R. Alonso et al. Summary report of MINSIS workshop in Madrid 2010. 1009.0476.

[13] S. Davidson, C. Peña-Garay, N. Rius and A. Santamaria. Present and future bounds on non-standard neutrino interactions. JHEP $\mathbf{0 3}$ 011, 2003. hep-ph / 0302093.

[14] J. Barranco, O. G. Miranda, C. A. Moura and J. W. F. Valle. Constraining non-standard interactions in nu/e e or anti-nu/e e scattering. Phys. Rev. D73 113001, 2006. hep-ph/ 0512195.

[15] J. Barranco, O. G. Miranda, C. A. Moura and J. W. F. Valle. Constraining Nonstandard Neutrino-Electron Interactions. Phys. Rev. D77 093014, 2008. 0711.0698.

[16] A. Bolanos et al. Probing non-standard neutrino-electron interactions with solar and reactor neutrinos. Phys. Rev. D79 113012, 2009. 0812.4417.

[17] C. Biggio, M. Blennow and E. Fernandez-Martinez. Loop bounds on non-standard neutrino interactions. JHEP 03 139, 2009. 0902.0607.

[18] S. Antusch, J. P. Baumann and E. Fernandez-Martinez. Non-Standard Neutrino Interactions with Matter from Physics Beyond the Standard Model. Nucl. Phys. B810 369, 2009. 0807.1003.

[19] M. B. Gavela, D. Hernandez, T. Ota and W. Winter. Large gauge invariant non-standard neutrino interactions. Phys. Rev. D79 013007, 2009. 0809.3451.

[20] M. S. Bilenky and A. Santamaria. One loop effective Lagrangian for a standard model with a heavy charged scalar singlet. Nucl. Phys. B420 47, 1994. hep-ph/9310302.

[21] S. Bergmann, Y. Grossman and D. M. Pierce. Can lepton flavor violating interactions explain the atmospheric neutrino problem? Phys. Rev. D61 053005, 2000. hep-ph/9909390.

[22] F. Cuypers and S. Davidson. Bileptons: Present limits and future prospects. Eur. Phys. J. C2 503, 1998. hep-ph/9609487.

[23] A. De Gouvea, G. F. Giudice, A. Strumia and K. Tobe. Phenomenological implications of neutrinos in extra dimensions. Nucl. Phys. B623 395, 2002. hep-ph/0107156.

[24] A. Broncano, M. B. Gavela and E. E. Jenkins. The effective Lagrangian for the seesaw model of neutrino mass and leptogenesis. Phys. Lett. $\mathbf{5 5 2}$ 177, 2003. hep-ph/0210271.

[25] S. Antusch, C. Biggio, E. Fernandez-Martinez, M. B. Gavela and J. Lopez-Pavon. Unitarity of the Leptonic Mixing Matrix. JHEP 10 084, 2006. hep-ph/ 0607020.

[26] A. Abada, C. Biggio, F. Bonnet, M. B. Gavela and T. Hambye. Low energy effects of neutrino masses. JHEP 12 061, 2007. 0707.4058.

[27] D. Tommasini, G. Barenboim, J. Bernabeu and C. Jarlskog. Non-decoupling of Heavy Neutrinos and Lepton Flavour Violation. Nucl. Phys. B444 451, 1995. hep-ph/ 9503228.

[28] E. Fernandez-Martinez, M. B. Gavela, J. Lopez-Pavon and O. Yasuda. CP-violation from non-unitary leptonic mixing. Phys. Lett. B649 427, 2007. hep-ph/ 0703098.

[29] S. Goswami and T. Ota. Testing non-unitarity of neutrino mixing matrices at neutrino factories. Phys. Rev. D78 033012, 2008. 0802.1434.

[30] S. Antusch, M. Blennow, E. Fernandez-Martinez and J. Lopez-Pavon. Probing non-unitary mixing and CP-violation at a Neutrino Factory. Phys. Rev. D80 033002, 2009. 0903.3986. 